[2] Azzollini J, Rovina D, Gervasini C, Parenti I, Fratoni A, Cubellis MV, et al. Functional characterisation of a novel mutation affecting the catalytic domain of MMP2 in siblings with multicentric osteolysis, nodulosis and arthropathy. J Hum Genet 2014 Nov;59(11):631-7.

[3] Castberg FC, Kjaergaard S, Mosig RA, Lobl M, Martignetti C, Martignetti $\mathrm{JA}$, et al. Multicentric osteolysis with nodulosis and arthropathy (MONA) with cardiac malformation, mimicking polyarticular juvenile idiopathic arthritis: case report and literature review. Eur J Pediatr 2013 Dec;172 (12):1657-63.

Disclosure of Interest: None declared

DOI: 10.1136/annrheumdis-2018-eular.2995

\section{THU0594 \\ SHIARI-JAVADI CRITERIA FOR THE DIAGNOSIS OF GENERALISED JOINT HYPERMOBILITY IN CHILDREN}

R. Shiari, V.J. Parvaneh. Pediatric Rheumatology, Shahid Beheshti University of Medical Sciences, Tehran, Iran, Islamic Republic of Ireland

Background: Benign joint hypermobility syndrome (BJHS) is the occurrence of musculoskeletal symptoms in hypermobile individuals in the absence of systemic rheumatologic disease. ${ }^{1-3}$ One of the most common criteria for the evaluation of generalised joint hypermobility is Beighton's criteria. However, Beighton criteria were designed for all ages not specifically for children group (according to the children physiological and growth characteristics). Inability to identify limited hypermobility, failure to differentiate generalised joint hypermobility from joint hypermobility syndrome and BJHS and the restrictions on the use of epidemiological studies are further limitations. ${ }^{4,5}$

Objectives: Because of the disadvantages of the existing criteria and the high prevalence of generalised joint hypermobility in children, we decided to propose further modifications to Beighton criteria for the diagnosis of joint hypermobility. Methods: A case-control study was designed with 200 participants from 3 to 16 years of age with 100 children with BJH (according to Beighton criteria) in case group and 100 age-sex matched children as control group. Cases were selected from outpatients Clinic of Rheumatology and the control group was selected from the emergency department of hospital. The case group consisted of children who had musculoskeletal pain complaints or were suspected to be hypermobile. The Beighton criteria were used as the gold standard, and all of cases fulfilled the Beighton criteria. In addition, the participants in the control group should be free of chronic disease or musculoskeletal complaints. For the determination of new criteria we performed literature review on the range of motion in different age and gender paediatric groups, studied the various existing criteria and their differences, and utilised our own clinical observations. Eight manoeuvres were set for examination (Fig 1). All were bilateral, except the neck hyperextension.

Results: There were $42(42 \%)$ male children in each group. The mean age was 6.8 years. Table 2 compares the results of the new and Beighton criteria in both cases and controls. All cases were hypermobile, and two of the 100 controls were hypermobile with the new criteria. Based on these results, new proposed criteria had the sensitivity, specificity, positive predictive value and negative predictive value of $100 \%, 98 \%, 100 \%$ and $98 \%$ respectively.
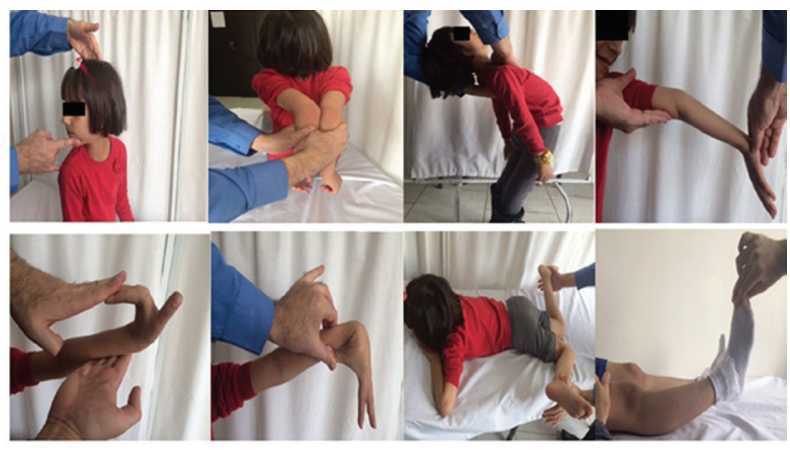

Conclusions: Shiari-Javadi criteria appears to be useful for detecting hyper mobility. In addition, they have also overcome many of the disadvantages of the Beighton criteria, and are easier, more practical and more comprehensive to be used in children. However, further studies are required to validate this criteria.

\section{REFERENCES:}

[1] Al-Rawi ZS, Al-Rawi ZT. Joint hypermobility in women with genital prolapse. Lancet 1982;1:1439-1441.

[2] Birrell FN, Adebajo AO, Hazleman BL, Silman AJ. High prevalence of joint laxity in West Africans. Br J Rheumatol 1994;33:56-59.
[3] Klemp P, Williams SM, Stansfield SA. Articular mobility in Maori and European New Zealanders. Rheumatology. 2002;41:554-557.

[4] Beighton PH, Grahame R, Bird HA, eds. In: Hypermobility of Joints 4th ed Berlin: Springer 2012.

[5] Ross J, Grahame R. Easily missed? Joint hypermobility syndrome. BMJ $2011 ; 342: 7167$

Acknowledgements: We would like to extend our sincer gratitude to all members of Paediatric Rheumatology Ward in Mofid Children Hospital and speciall thanks to Miss Elham Musavi.

Disclosure of Interest: None declared

DOI: 10.1136/annrheumdis-2018-eular.7612

\section{THU0595 CLINICAL AND THERAPEUTICAL DIFFERENCES BETWEEN ADULTS AND CHILDREN IN A COLOMBIAN} COHORT WITH INFLAMMATORY IDIOPATIC MYOPATHY

R. Fuentes ${ }^{1}$, M. Cañola ${ }^{1}$, J.C. Diaz-Coronado ${ }^{2}$, A. Rojas-Villarraga ${ }^{2}$,

D. Hernandez-Parra ${ }^{2}$, P. Perez-Estrada ${ }^{2}$, J.C. Salazar-Uribe ${ }^{3}$, R. Pineda-Tamayo ${ }^{2}$ ${ }^{1}$ Rheumatology Department, ${ }^{2}$ Clinical Research, Artmedica; ${ }^{3}$ National University of Colombia, Medellin, Colombia

Background: Age-related autoimmunity is a key differentiating factor with diverse presentations in different age groups possibly depending on immune regulatory mechanisms. Even patients with the same diagnostic category may have different presentations according to age and other factors. Inflammatory Idiopathic Myopathies (IIM) are not the exception with important clinical and prognostic differences between adults and children.

Objectives: To describe the clinical and therapeutical differences between adults and children (younger tahn $18 \mathrm{~m}$ years old) in a cohort with IIM

Methods: A cross-section retrospective research was done with data collected between 2014 and 2017 from a population diagnosed with IIM according to Peter and Bohan criteria and followed up for at least six months. Statistical association was evaluated by means of Chi-square and Fisher's tests.

Results: Out a total of 149 patients, 112 were adults and 37 were children. There was significant difference between the clinical presentation of symmetrical muscle weakness $98 \%$ in adults vs $73 \%$ in children (p: 0,0001 ), Gottron's papules $32 \%$ vs $89 \%$ (p: 0,0001 ), Heliotrope rash $31 \%$ vs $62 \%$ (p: 0,0016 ), Calcinosis cutis $1 \%$ vs $37 \%$ (p: 0,0001), Dermatological involvement 47\% vs $94 \%$ (p: 0,0001), Myopathic changes in the electromyogram $41 \%$ vs $25 \%$ (p: 0,0001), and Myopathy proven biopsy $41 \%$ vs $25 \%$ (p: 0,0001 ). Theraepeutical options showed a significant difference in the use of steroid therapy $93 \%$ vs. $84 \%$ (p: 0,045), Methotrexate $(58 \%$ vs $91 \%$ p: 0,0001), Azatioprine (62\% vs $27 \%$ p: 0,0002$)$ and Cloroquine ( $31 \%$ vs $73 \%$ p: 0,0001). Other therapies like Cyclophosphamide (19\% vs 5\% p: 0,04) and Inmunoglobulins ( $23 \%$ vs $5 \%$ p: 0,01$)$ were significantly different between adults and children.

Abstract THU0595 - Table 1. Clinical characteristics of Colombian Patients with Inflammatory Idiopathic Myopath (Adults and Children)

\begin{tabular}{lccccc}
\hline & \multicolumn{2}{c}{$\begin{array}{c}\text { Juvenile } \\
\mathbf{n = 3 7}\end{array}$} & \multicolumn{2}{c}{$\begin{array}{c}\text { Adults } \\
\mathbf{n = 1 1 2}\end{array}$} & \\
& $\mathbf{N}$ & $\%$ & $\mathbf{N}$ & $\%$ & $\begin{array}{c}\mathbf{p}- \\
\text { value }\end{array}$ \\
\hline Female & 28 & 75,7 & 76 & 67,9 & 0,42 \\
Clinical characteristics & & & & & \\
Symmetrical muscle weakness & 27 & 73 & 110 & 98,2 & $<, 0001$ \\
Gottron's papules & 33 & 89,2 & 49 & 32,9 & $<, 0001$ \\
Heliotrope rash & 23 & 62,2 & 35 & 31,3 & $\mathbf{0 , 0 0 1 6}$ \\
Calcinosis cutis & 14 & 37,8 & 2 & 1,8 & $<, 0001$ \\
Dermatological involvement & 35 & 94,6 & 53 & 47,3 & $<, 0001$ \\
Myophatic changes (EMG) & $9 / 23$ & 39,1 & $68 /$ & 84,0 & $<, 0001$ \\
& & & 81 & & \\
Biopsy-proven myopathy & $4 / 16$ & 25 & $46 /$ & 41,1 & $<, 0001$ \\
(Positive) & & & 54 & & \\
ANA(+) & $14 /$ & 51,9 & $65 /$ & 73,0 & $\mathbf{0 , 0 5 8 0}$ \\
& 27 & & 89 & & \\
\hline
\end{tabular}

\section{EMG, electromyogram}

Conclusions: Several differences in the clinical and therapeutical characeristics were found between adults and children with IIM. Adults had more frequently symmetrical weakness, myopathic changes in EMG and biopsy proven myopathy. In contrast children had higher skin involvement. Calcinosis constituded an important manifestation of IIM in children. These results suggest that paediatric IIM is a distinct sub-phenotype related to the early age at onset and possibly mediated by different immune interplay key factors. 


\section{REFERENCE:}

[1] Tansley S, et al. Comparing and contrasting clinical and serological features of juvenile and adult-onset myositis: implications for pathogenesis and outcomes.1040-8711 Copyright! 2015 Wolters Kluwer Health, Inc

Disclosure of Interest: None declared

DOI: 10.1136/annrheumdis-2018-eular.4861

\section{THU0596 MEASUREMENT OF SERUM CALPROTECTIN MRP8/14 IN PAEDIATRIC PATIENTS DIAGNOSED WITH JUVENILE IDIOPATHIC ARTHRITIS AND AUTOINFLAMMATORY DISEASES}

S. Murias ${ }^{1}$, C. Udaondo ${ }^{1}$, R.M. Alcobendas ${ }^{1}$, A. Remesal ${ }^{1}$, D. Pascual-Salcedo ${ }^{2}$. ${ }^{1}$ Pediatric Rheumatology; ${ }^{2}$ Immunology Department, University Hospital La Paz, Madrid, Spain

Background: There is evidence of the correlation between serum levels of calprotectin MRP8/14 and disease activity in Juvenile Idiopathic Arthritis (JIA), but the same correlation with other rheumatic diseases in children such as autoinflammatory diseases has not been studied as much.

Objectives: To analyse calprotectin MRP8/14 in serum of patients with juvenile idiopathic arthritis (JIA) and various autoinflammatory diseases with different degrees of activity. To check if there is a correlation between serum MRP8/14 and disease activity.

Methods: Study in two phases: 1) Initial, transversal, in JIA and/or autoinflammatory disease patients, determining serum MRP8/14 (by ELISA test) and collecting clinical data: number of active joints, Visual Analogue Scale parents/patients (VASp), VAS physician (VASph); and analytical: C-Reactive Protein (CPR) and white cells blood count (WBC) (among others). 2) Second prospective phase, repeating the same analysis 3-6 months later. Disease activity was assessed by Juvenile Arthritis Disease Activity Score (JADAS) in AIJ; and by VASp, VASph and CRP in autoinflammatory diseases.

Results: The sample included 90 patients ( 25 males) between the ages of 1 and 21 years (median 11.1); 61 diagnosed with JIA (excluding systemic category), and 29 with different autoinflammatory diseases (including systemic JIA). 69 patients (20 males, with median age 11.2, 48 AlJ, 21 autoinflammatory) participated in the prospective phase. The main results are shown in table 1.

$\mathrm{MRP}=$ myeloid related proteins. JADAS=Juvenile arthritis disease activity score. $A C J=a c t i v e$ joints count. VASm=visual analogue scale-physician. VASp=visual analogue scale-parents/patient. $\mathrm{CRP}=\mathrm{C}$ reactive Protein.

Conclusions: In patients with non-systemic JIA, a positive correlation was found between serum MRP8/14 and JADAS, VASph, VASp, CRP, WBC, neutrophilia and (inversely) haemoglobin.

In autoinflammatory diseases positive correlation has been found with VASm, CRP, leukocytosis, neutrophilia, thrombocytosis and (inversely) haemoglobin. MRP8/14 seems to behave as a marker of activity in both JIA and autoinflammatory diseases, although with a stronger association in the latter.

Disclosure of Interest: None declared

DOI: 10.1136/annrheumdis-2018-eular.4526

\section{THU0597}

PULMONARY MANIFESTATIONS IN MIXED CONNECTIVE TISSUE DISEASE WITH JUVENILE AND ADULT ONSET - ARE THERE ANY DIFFERENCES?

S.O. Hetlevik ${ }^{1,2}$, S. Reiseter ${ }^{1}$, Ø. Molberg ${ }^{1,2}$, B. Flatø ${ }^{1,2}$, R. Gunnarsson ${ }^{1}$, T. M. Aaløkken ${ }^{3}$, M.B. Lund ${ }^{4}$, V. Lilleby ${ }^{1}{ }^{1}$ Rheumatology, Oslo University Hospital; ${ }^{2}$ Faculty of medicine, University of Oslo; ${ }^{3}$ Department of radiology and nuclear medicine; ${ }^{4}$ Department of respiratory medicine, Oslo University Hospital, Oslo, Norway

Background: Mixed connective tissue disease (MCTD) presents in childhood in $7 \%-23 \%$ of cases, but there is limited knowledge about the comparability of juvenile and adult onset of the disease. A common and serious manifestation is interstitial lung disease (ILD), possibly more common in adult MCTD according to a retrospective report[.

Objectives: To compare disease variables in juvenile and adult onset MCTD, particularly regarding pulmonary manifestations, after long-term follow-up.

Methods: Two cohorts consisting of, respectively, 52 patients with juvenile onset MCTD and 90 patients with adult onset MCTD from all regions of Norway were compared. Inclusion criteria were fulfilment of the Kasukawa- or the AlarconSegovia criteria. Patients with onset of symptoms before the age of 18 years were considered to have juvenile onset. All patients were clinically examined, including high resolution $\mathrm{CT}$ and pulmonary function tests.

Results: Mean age at examination was 28.0 (SD 10.3) in juvenile onset, and 54.3 (SD 13.0) in adult onset MCTD ( $p<0.001)$. Age at disease onset was 14.4 (SD4.4) and 37.9 (SD 15.1) years, respectively. Patient and disease characteristics are shown in table 1.

\begin{tabular}{lccc}
\hline & Juvenile onset & Adult onset & $\begin{array}{c}p \text { - } \\
\text { value }\end{array}$ \\
\hline Male gender, n (\%) & $8(15)$ & $28(24)$ & 0.192 \\
Disease duration from symptom onset, mean & $16.2(10.3)$ & $17.0(8.9)$ & 0.766 \\
(SD) & & & \\
Anti-RNP levels, median (IQR) & $180(36.0-$ & $44.0(7.0-$ & 0.002 \\
& $240.0)$ & $237.0)$ & \\
ESR, median (IQR) & $8.0(5.0$ to & $11.0(7.0-18.5)$ & 0.048 \\
& $16.0)$ & & \\
CRP, median (IQR) & $0.7(0.5-2.5)$ & $2.0(0.7-4.9)$ & $<0.001$ \\
ILD, n (\%) & $14(27)$ & $39(43)$ & 0.051 \\
ILD\% of TLV, median (IQR) & $4.0(1.0-8.3)$ & $5.0(1.8-20.0)$ & 0.258 \\
ILD in>20\% of TLV, n (\%) & $1(2)$ & $10(11)$ & 0.048 \\
DLCO\% pred, mean (SD) & $73.0(12.5)$ & $72.0(19.4)$ & 0.598 \\
FVC\% pred, mean (SD) & $89.2(14.7)$ & $89.2(20.8)$ & 0.711 \\
FEV1\% pred, mean (SD) & $89.4(13.7)$ & $81.2(20.0)$ & 0.016 \\
\hline
\end{tabular}

Juvenile patients had higher levels of anti-RNP, but lower ESR and CRP compared to adult onset patients. ILD was found in $27 \%$ of juvenile and $43 \%$ of adult patients $(p=0.051)$, and more adult onset patients had ILD in $>20 \%$ of total lung volume (TLV). Adult onset patients had lower forced expiratory volume in $1 \mathrm{~s}$ (FEV1), but similar diffusing capacity (DLCO) and forced vital capacity (FVC) as the juvenile patients.

Conclusions: ILD tended to be more frequent in patients with adult onset MCTD than in those with juvenile onset, although not statistically significant. More patients with adult onset had affection of $>20 \%$ of TLV. Juvenile and adult patients

\begin{tabular}{|c|c|c|c|c|}
\hline & \multicolumn{2}{|c|}{ JIA $(n=61)$} & \multicolumn{2}{|c|}{ Autoinflammatory diseases $(n=29)$} \\
\hline & $\begin{array}{c}1 \text { st determination } \\
(n=61)\end{array}$ & $\begin{array}{l}\text { 2nd determination } \\
\qquad(\mathrm{n}=48)\end{array}$ & $\begin{array}{c}1 \text { st determination } \\
(n=29)\end{array}$ & $\begin{array}{l}\text { 2nd determination } \\
\qquad(\mathrm{n}=21)\end{array}$ \\
\hline JADAS & $r=0.270^{*} \mathrm{p}=0.035$ & $\begin{array}{l}r=0.054 \\
p=0.717\end{array}$ & & \\
\hline ACJ & $\begin{array}{l}r=0.053 \\
\mathrm{p}=0.685\end{array}$ & $\begin{array}{l}r=0.042 \\
p=0.779\end{array}$ & & \\
\hline VASph & $r=0.308^{*} \mathrm{p}=0.016$ & $\begin{array}{l}r=0.041 \\
p=0.784\end{array}$ & $\begin{array}{l}r=0.304 \\
p=0.108\end{array}$ & $r=0.622^{* *} \mathrm{p}=0.003$ \\
\hline VASp & $r=0.329^{* *} \mathrm{p}=0.010$ & $\begin{array}{l}r=-0.023 \\
p=0.877\end{array}$ & $\begin{array}{l}r=0.341 \\
p=0.082\end{array}$ & $\begin{array}{l}r=0.341 \\
\mathrm{p}=0.141\end{array}$ \\
\hline CRP & $\begin{array}{l}r=0.212 \\
p=0.107\end{array}$ & $r=0.349^{*} \mathrm{p}=0.018$ & $r=0.579^{\star *} \mathrm{p}=0.001$ & $\begin{array}{l}r=0.102 \\
\mathrm{p}=0.688\end{array}$ \\
\hline WBC & $r=0.296^{*} \mathrm{p}=0.022$ & $\begin{array}{c}r=-0.071 \\
\mathrm{p}=0.633\end{array}$ & $r=0.749^{* *} \mathrm{p}=0.000$ & $r=0.693^{\star *} \mathrm{p}=0.000$ \\
\hline Neutrophiles & $r=0.389^{* \star} \mathrm{p}=0.002$ & $r=0.421^{\star \star} \mathrm{p}=0.002$ & $\begin{array}{l}r=0.281 \\
\mathrm{p}=0.140\end{array}$ & $r=0.710^{\star \star} \mathrm{p}=0.000$ \\
\hline Haemoglobin & $r=-0.255^{\star} \mathrm{p}=0.049$ & $\begin{array}{c}r=-0.023 \\
\mathrm{p}=0.828\end{array}$ & $r=-0.454^{*} \mathrm{p}=0.013$ & $r=-0.552^{* *} \mathrm{p}=0.009$ \\
\hline Platelets & $\begin{array}{l}r=-0.033 \\
\mathrm{p}=0.802\end{array}$ & $\begin{array}{l}r=0.001 \\
\mathrm{p}=0.993\end{array}$ & $r=0.659^{* *} \mathrm{p}=0.000$ & $\begin{array}{l}r=0.208 \\
\mathrm{p}=0.366\end{array}$ \\
\hline
\end{tabular}

